\title{
Dynamische lumbale Techniken: Erwartungen und Evidenz
}

Michael Stoffel

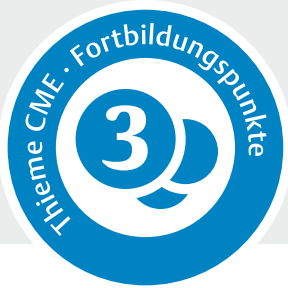

\section{Einleitung}

Ein spinales Bewegungssegment ist die kleinste funktionelle Einheit an der Wirbelsäule. Es besteht aus:

- 2 angrenzenden Wirbeln,

- der dazwischen liegenden Bandscheibe,

- den Facettengelenken,

- den umliegenden Bändern und Muskeln.

Ein entscheidender Vorgang bei der Degeneration an der Wirbelsäule ist die Degeneration und zunehmende Höhenminderung der Bandscheibe. Dadurch ändert sich die Biomechanik im Bewegungssegment dergestalt, dass der Bewegungsumfang bei Krafteinwirkung

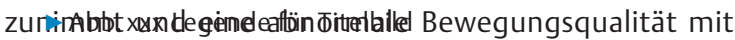
abnormaler Kraftverteilung zwischen Bandscheibenraum und Facettengelenken entsteht [1]. Wenn dieser pathologisch erhöhte Bewegungsumfang in einem Bewegungssegment zu Beschwerden führt (belastungsabhängiger Rückenschmerz und/oder pseudoradikulär ausstrahlender Schmerz), spricht man von klinisch relevanter Instabilität.

\section{Merke}

Ein spinales Bewegungssegment ist die kleinste funktionelle Einheit an der Wirbelsäule. Es besteht aus 2 angrenzenden Wirbeln, der dazwischen liegen-

$\begin{array}{ll}\text { ABKÜRZUNGEN } \\ \text { ASD } & \text { Anschlusssegmentdegeneration } \\ \text { DDD } & \text { degenerative disc disease } \\ \text { IPD } & \text { interspinous process device } \\ \text { ODI } & \text { Oswestry Disability Index } \\ \text { PDN } & \text { prosthetic disc nucleus } \\ \text { PDS } & \text { Pedikelschrauben-basierte dynamische } \\ & \text { Systeme } \\ \text { PLIF } & \text { posteriore lumbale interkorporelle Fusi- } \\ & \text { on } \\ \text { RCT } & \text { randomized controlled trial } \\ \text { ROM } & \text { range of motion } \\ \text { TDR } & \text { total disc replacement } \\ \text { VAS } & \text { visuell-analoge Schmerzskala } \\ \text { ZCQ } & \text { Zürich Claudicatio Questionnaire }\end{array}$

den Bandscheibe, den Facettengelenken und den umliegenden Bändern und Muskeln.

Neben degenerativen Veränderungen kann auch die operative Dekompression (mediale Facettektomie, Laminektomie usw.), z. B. zur Behandlung eines Bandscheibenvorfalls oder einer Spinalkanalstenose, zur Zunahme des Bewegungsumfangs und zur klinischen Instabilität führen. Dies betrifft grundsätzlich alle Bewegungsrichtungen (Flexion/Extension, Seitwärtsbeugen, Rotation), ist aber für die Rotation am stärksten ausgeprägt [2].

Merke

Klinisch relevante Instabilität kann durch Degeneration der Bandscheibe mit Höhenminderung oder durch operative Verfahren mit Gelenkbeteiligung verursacht oder begünstigt werden.

Da bei der klinischen Instabilität die pathologische Zunahme der Beweglichkeit zu Schmerzen führt, hat sich seit der Mitte des 20. Jahrhunderts die Spondylodese (Synonyme: rigide Stabilisierung, Fusion, Versteifung) als Standardtherapie etabliert. Hier existieren inzwischen verschiedene Techniken mit akzeptablen klinischen Ergebnissen (siehe Hintergrundbox). Die Nachteile der Fusion ergeben sich aus dem recht hohen operativen Aufwand, bei dem die Inzidenz und Schwere der Komplikationen mit zunehmender Komplexität steigen, dem Pseudarthroserisiko und aus dem Transfer von Stress auf die benachbarten Bewegungssegmente, deren Degeneration dadurch beschleunigt wird (Anschlusssegmentdegeneration). Mehr als $1 / 3$ der Patienten leidet 10 Jahre nach lumbaler Spondylodese unter einer symptomatischen Anschlusssegmentdegeneration (ASD) $[3,4]$.

\section{HINTERGRUND}

Merkmale der lumbalen Spondylodese

- Vielzahl chirurgischer Techniken (dorsolaterale Fusion, ALIF, XLIF, TLIF usw.)

- akzeptable Ergebnisse (mean $\triangle$ ODI: 20)

- keine spezielle Technik allgemein überlegen

- Inzidenz/Schweregrad von Komplikationen $\uparrow$ mit zunehmender Komplexität 
Da andererseits die Fusionsrate (ca. 90\%) nicht mit der Rate an klinisch erfolgreicher Schmerzlinderung (ca. $70 \%$ ) bei Patienten nach Spondylodese korreliert, scheint das komplette Stilllegen eines Bewegungssegments nicht zwingende Voraussetzung für die Beschwerdelinderung zu sein. Aus diesen Überlegungen heraus haben sich die dynamischen Verfahren (Synonym: Non-Fusion-Techniken) an der Wirbelsäule etabliert mit folgenden Zielen:

- Beheben von Schmerz, verursacht durch geschädigtes Bewegungssegment,

- Erhalt oder Wiedererlangen von Stabilität,

- Erhalt von Mobilität und Funktion der Wirbelsäule,

- Langzeitziel: Stressminimierung auf Anschlusssegment durch Bewegungserhalt im Indexsegment.

Die dynamischen Verfahren können unterteilt werden in:

- Gelenkersatzverfahren (Synonyme: Arthroplastie, Prothetik), bei denen entweder nur der Nucleus pulposus (nucleus replacement) oder die ganze Bandscheibe (total disc replacement, TDR) entfernt und ersetzt werden,

- dynamische Stabilisierungsverfahren, bei denen von dorsal eingebrachte Implantate den Bewegungsumfang des Bewegungssegments reduzieren und zu einer Umverteilung der Krafteinwirkung zwischen Bandscheibe und Facettengelenken führen (load sharing). Hier werden die Pedikelschraubenbasierten dynamischen Systeme (PDS) von den interspinösen Spreizern (interspinous process devices, IPDs) unterschieden.

Inzwischen findet sich noch eine dritte Gruppe - die Facettengelenkersatzverfahren - in der frühen Phase der Entwicklung und ersten Anwendung. Diese Verfahren können sowohl als Gelenkersatzverfahren als auch als Pedikelschrauben-basierte dynamische Systeme gesehen werden und unter beiden Verfahrensgruppen abgehandelt werden.

\section{Dynamische Verfahren}

\section{Nucleus-pulposus-Ersatz (nucleus replacement)}

\section{Prinzip, Historie und Indikation}

Das Behandlungsprinzip der Nukleusersatzverfahren besteht aus dem:

- Wiederherstellen der Bandscheibenhöhe und der Anulus-fibrosus-Spannung,

- Erhalt der Segmentflexibilität unter physiologischer Krafteinwirkung.
Der Nucleus pulposus wird ersetzt über einen „minimalinvasiven“ Zugang, bei dem der Anulus fibrosus größtenteils intakt gelassen wird. Damit sollten diskogene Pathologien adressiert werden und die therapeutische Lücke zwischen ausschließlicher Diskektomie und Stabilisierung geschlossen werden.

\section{THERAPIEZIELE}

\section{Nucleus-pulposus-Ersatz}

Ziel ist die Reduktion von diskogenem Schmerz, der fortschreitenden Progression der Segmentdegeneration und das Wiedererlangen „nicht degenerativer“ spinaler Mobilität.

Nach Vorläufern, wie der Metallkugel, die von Fernström zum Ersatz der degenerierten Bandscheibe eingesetzt worden ist, waren Bao u. Mitarb. die ersten, die ein Hygrogelmaterial benutzten und damit erstmals eine „echte“ Nukleusprothese geschaffen haben $[5,6]$.

Als Indikation wurde die monosegmentale, lumbale, symptomatische Bandscheibendegeneration in noch recht frühem Stadium (Pfirrmann 2, 3, Bandscheibenhöhe $>5 \mathrm{~mm}$ ) bei intakten Facettengelenken und konservativer Therapierefraktärität (>6 Monate) beschrieben [7].

Kontraindikationen sind:

- Body Mass Index >30,

- Bandscheibenhöhenverlust von mehr als $50 \%$,

- Schmorl-Knötchen der Endplatten,

- Infektionen,

- Osteoporose,

- Spondylolisthesen (> Grad I nach Meyerding).

\section{Klinische Ergebnisse}

Der Prosthetic Disc Nucleus (PDN, Raymedica, Minneapolis, MN) ist das weltweit am meisten eingesetzte und untersuchte Implantat. Ähnlich wie der Nucleus pulposus selbst absorbiert der PDN Wasser bis zu $90 \%$ seines Trockengewichts nach Implantation und dehnt sich somit aus. Da es, wie alle späteren Nukleusimplantate auch, nicht verankert wird, waren Materialdislokation und Einbruch in Grund- und Deckplatten (subsidence) die Hauptprobleme. Durch einige Änderungen in der Implantatgeometrie konnten die klinischen Erfolgsraten zwischen der ersten Phase-I-Studie und einer Phase-IV-Studie von $62 \%$ auf $91 \%$ - jeweils in kleinen Patientenkohorten - verbessert werden [8].

2002 erschienen von Klara u. Ray und von Bertagnoli u. Schönmayr Ergebnisse mit bis zu 4 Jahren Nachbeobachtung, die einen signifikanten und anhaltenden Effekt von PDN auf Oswestry Disability Index (ODI), visu- 
ell-analoge Schmerzskala (VAS), Prolo-Score und Bandscheibenhöhe zeigen konnten $[9,10]$. Nichtsdestotrotz haben sich diese Implantate nie in Deutschland als wesentlicher Bestandteil des Prozedurenarmamentariums bei der DDD durchgesetzt.

\section{Arthroplastie}

Prinzip, Historie und Indikation

\section{THERAPIEZIELE}

\section{Bandscheibenarthroplastie}

Ziel der Bandscheibenarthroplastie (Synonyme: Bandscheibenprothetik, total disc replacement, TDR) ist das Entfernen der schmerzgenerierenden Strukturen der Bandscheibe und das Wiederherstellen der ehemaligen Bandscheibenhöhe, deren Winkel und Funktion.

Die Anfänge des beweglichen Bandscheibenersatzes liegen in den 1950er-Jahren und waren von frustranen Ergebnissen geprägt. Einen ganz entscheidenden Entwicklungsschritt stellt die SB-Charité-Prothese dar, die von Schellnac u. Büttner-Janz 1982 entwickelt und von Zippel 1984 erstmals implantiert wurde. Sie bestand aus einem beweglichen Kern aus Polyethylen (UHMWPE = ultra-high-molecular weight polyethylene) zwischen metallenen Endplatten. Anfängliche Probleme durch Materialmigration und -ermüdung haben zu Weiterentwicklungen und seit 1987 zur SB Charité III geführt [11], die inzwischen über 7000 Mal weltweit implantiert und in vielen Studien evaluiert worden ist.

Inzwischen stehen Prothesen mit 3 verschiedenen Designs zur Verfügung ( $\triangleright$ Tab. 1):
- Modulare 3-Komponenten-Prothesen (Metallendplatten/Kunststoffkern),

- Metal-on-Metal-Prothesen (Ball-and-SocketPrinzip),

- Compressible-Core-Prothesen.

Sie werden kinematisch kategorisiert als constrained, semiconstrained oder unconstrained entsprechend ihrer Fähigkeit, segmentale Bewegung zu begrenzen oder zuzulassen verglichen mit normalen physiologischen Parametern ( $\triangleright$ Tab. 1).

Einzige Indikation zur Implantation einer lumbalen Bandscheibenprothese ist der therapierefraktäre diskogene Kreuzschmerz, bei dem die degenerativen Veränderungen in der Bandscheibe (degenerative disc disease, DDD) zur intradiskalen Entstehung von Schmerzen führen. Der intradiskale Schmerz ist abzugrenzen von einem Schmerz, der im Facettengelenk entsteht und durch Facettengelenkinfiltration transient gelindert oder aufgehoben werden kann.

Ein im Vordergrund stehender „Facettengelenk"schmerz stellt eine Kontraindikation für eine Bandscheibenprothese dar.

\section{Cave}

Die degenerative Bandscheibenerkrankung (DDD) mit therapierefraktärem diskogenen Kreuzschmerz ist die einzige Indikation zur lumbalen Bandscheibenprothese. Signifikanter „Facettengelenk“schmerz stellt eine Kontraindikation dar.

\section{Der „typische“ Patient}

Für eine Bandscheibenprothese kommen nur Patienten infrage mit „diskogenem“ Rückenschmerz, also einem Schmerz, der in der Bandscheibe entsteht. Dieser ist häufig durch Rumpfrotation und Flexion zu provozieren

- Tab. 1 Auswahl verschiedener lumbaler Prothesen.

\begin{tabular}{|c|c|c|c|c|}
\hline Produkt & Hersteller & Kinematik & Biomechanik & Biomaterialien \\
\hline Charité & DePuy Spine, Rahnham, MA & unconstrained & Metall/Polymer & CoCrMo-Endplatten, UHMWPE-Kern \\
\hline M6-L & Spinal Kinetics, Sunnyvale, CA & unconstrained & Metall/Polymer & $\begin{array}{l}\text { Titan-Hülle, Polycarbonaturethan und } \\
\text { Polyethylen-Anulus (gewebt) }\end{array}$ \\
\hline Acroflex & DePuy Spine, Rahnham, MA & unconstrained & Metall/Polymer & Titan-Endplatten, Polyolefin-Kern \\
\hline Mobidisc & $\begin{array}{l}\text { Mobidisc Lumbar Disc, LDR USA, } \\
\text { Austin, Tx }\end{array}$ & unconstrained & Metall/Polymer & CoCrMo-Endplatten, UHMWPE-Kern \\
\hline Prodisc-L & $\begin{array}{l}\text { DePuySynthes Spine, West } \\
\text { Chester, PA }\end{array}$ & semiconstrained & Metall/Polymer & CoCrMo-Endplatten, UHMWPE-Kern \\
\hline Maverick & Medtronic, Memphis, TN & semiconstrained & Metal-on-Metal & CoCrMo \\
\hline Flexicore & Stryker Spine, Allendale, NJ & constrained & Metal-on-Metal & CoCrMo \\
\hline
\end{tabular}


und muss abgegrenzt werden von dem Schmerz, der vornehmlich in den Facettengelenken entsteht. Eine Facettengelenkinfiltration oder auch eine Diskografie können die Differenzierung erleichtern $[12,13]$.

Die DDD sollte mono- oder bisegmental sein mit eindeutigen radiologischen Veränderungen der Bandscheibe (Höhenminderung, Abflachung des Lordosewinkels, intradiskale Signalveränderungen nach Pfirrmann, Grund- und Deckplattenosteochondrose nach Modic). High Intensity Zones - also dorsal im Faserring gelegene Aufhellungen in T2-werden ebenso mit intradiskalem Schmerz in Verbindung gebracht. Die Patienten sollten schlank, jünger als 50-55 Jahre alt mit noch suffizienter autochthoner Muskulatur und normaler Knochendichte sein und konservativ therapierefraktär (\Abb. 1).

\section{Klinische Ergebnisse}

\section{Bandscheibenarthroplastie versus Reha}

In einer prospektiv randomisierten Multicenterstudie der Norwegian Spine Study Group zeigten Patienten (Kreuzschmerz $>1$ Jahr, ODI $\geq 30$, DDD mono-/bisegmental) 2 Jahre nach ProDisc-II-Implantation eine signifikant geringere rückenschmerzassoziierte Behinderung (primärer Endpunkt, gemessen mit dem ODI) im Vergleich zur Kontrollgruppe (12 - bis 15-tägige multidisziplinäre Rehabilitation). Allerdings wurde die vorab spezifizierte Grenze von 10 Punkten Unterschied im ODI als „minimally important clinical difference“ nicht erreicht.

Folgende sekundäre Endpunkte zeigten zusätzlich einen signifikanten Unterschied zugunsten der TDR:
- Patientenzufriedenheit,

- SF-36 Physical Component,

- Self Efficacy for Pain,

- Prolo-Score.

Keine signifikanten Unterschiede ergaben sich bei:

- EQ-5D,

- Return-to-Work,

- SF-36 Mental Component,

- Fear Avoidance Beliefs,

- Hopkins Symptom Check List,

- Schmerzmitteleinnahme,

- Back Performance Scale.

In der Prothesengruppe führte eine schwere Komplikation bei einem Revisionseingriff wegen einer Polyethylenkern-Dislokation zu einer Beinamputation [14].

Die Patienten beider Behandlungsgruppen (operativ vs. konservativ) zeigten 2 Jahre nach Studieneinschluss Anschlusssegmentdegeneration mit gleicher Häufigkeit [15].

\section{Bandscheibenarthroplastie versus Fusion}

Ein Cochrane Review (2012) fasst die Ergebnisse von 6 prospektiv randomisierten Multicenterstudien zusammen [16]. Dabei zeigt sich nach 2 Jahren ein signifikant besseres Ergebnis des Rückenschmerzes (nach VAS), des ODI und der Patientenzufriedenheit, allerdings unterhalb des klinisch relevanten Unterschieds (clinically relevant difference). Trotz Sponsoring und fehlender Verblindung wird das Bias-Risiko der Studien als gering eingeschätzt.
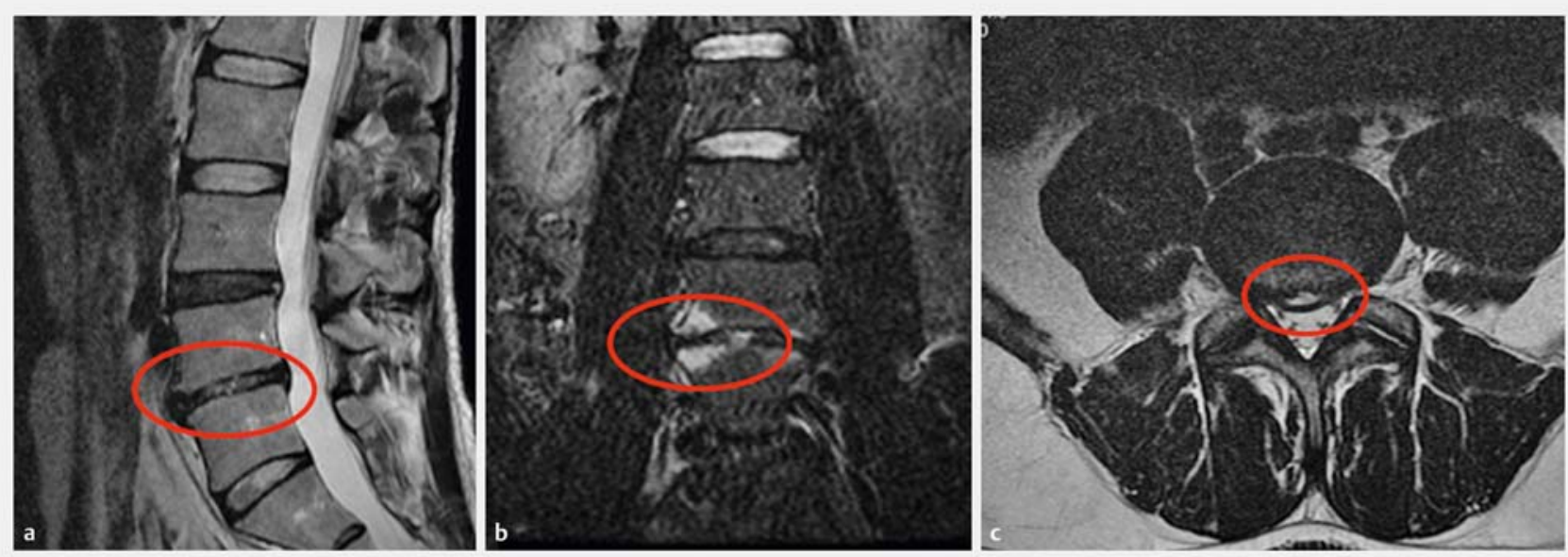

Abb. 1 a - c TDR - der typische Patient. Alter< 55 Jahre. Lumbaler Kreuzschmerz als Hauptsymptom. DDD: Bandscheiben-Höhenminderung $>40 \%$, Bandscheibendegeneration Pfirrmann 3 oder 4, Grund-/Deckplattenveränderungen Modic Typ I, II, dorsale High Intensity Zone, ODI $>20$ (30). Konservativ therapierefraktär. 
Eine Metaanalyse von 2013 bestätigt die Überlegenheit der Bandscheibenprothese zur Behandlung des Rückenschmerzes (VAS). Die Rate an Komplikationen war mit 5,8\% niedriger als nach Fusion (10,8\%). Reoperationen waren mit 5,2\% (TDR) bzw. 6\% (Fusion) vergleichbar häufig notwendig. Das Bewegungsausmaß (range of motion, ROM) war nach TDR im normalen Bereich geblieben [17].

Merke

Prothesendesign hat laut Studienlage keinen Einfluss auf das klinische Ergebnis.

\section{Bandscheibenarthroplastie: mittel- bis langfristige Ergebnisse}

Die meisten prospektiven, randomisierten Studien umfassen einen Nachbeobachtungszeitraum von 2 Jahren, was u.a. an den Forderungen der US-amerikanischen Zulassungsbehörde FDA liegt. Siepe u. Mitarb. haben prospektiv die Ergebnisse von 181 Patienten nach Prodisc-II-Implantation gesammelt (Nachbeobachtung: 5-10,8 Jahre). Dabei zeigt sich, dass die signifikante Verbesserung des Rückenschmerzes (VAS) und des ODI postoperativ über die gesamte Nachbeobachtung erhalten bleibt, ebenso wie die Patientenzufriedenheit (86,3\% zufrieden/hoch zufrieden) bei einer Komplikationsrate von 14,4\% und einer Revisionsrate von 7,2\% im gesamten Nachbeobachtungszeitraum [18].

FAZIT

Zusammenfassend zeigt sich eine Überlegenheit der bewegungserhaltenden Bandscheibenprothetik im kurzfristigen Vergleich (2 Jahre) zur konservativen Therapie mit Rehabilitation und zur Spondylodese (Level-I-Evidenz). Die klinischen Ergebnisse zeigen sich mittelfristig (5-10 Jahre) stabil (Level-II-2-Evidenz).

\section{Dynamische Stabilisierung}

\section{THERAPIEZIELE}

Dynamische Stabilisierung

Ziel der dynamischen Stabilisierungstechniken ist, durch von dorsal eingebrachte Implantate die abnormale Kraftverteilung auf Bandscheibe und Facettengelenke infolge der Segmentdegeneration zu reduzieren („load sharing”) unter Erhalt einer Restbeweglichkeit. Die Bandscheibe wird intakt gelassen.

\section{ZUSATZINFO}

Evidenzqualität entsprechend der U.S. Preventive Services Task Force

- Level I: Evidenz aus mindestens einer gut gestalteten randomisierten, kontrollierten Studie

- Level II-1: Evidenz aus gut gestalteten kontrollierten Studien ohne Randomisierung

- Level II-2: Evidenz aus gut gestalteten Kohorten- oder Fallkontrollstudien, vorzugsweise aus mehr als einem Zentrum oder Forschungsgruppe

- Level II-3: Evidenz aus mehreren Zeitreihenanalysen mit oder ohne Intervention. Tiefgreifende Ergebnisse aus unkontrollierten Studien können auch als Level II-3 angesehen werden

- Level III: Meinungen respektierter Persönlichkeiten, basierend auf klinische Erfahrung, beschreibende Studien oder Berichte von Expertenkomitees

Man unterscheidet:

- Pedikelschrauben-basierte Verfahren,

- interspinöse Spreizer,

- Facettengelenkersatzverfahren.

Da der operative Schritt des Ausräumens der Bandscheibe und des Füllens mit Fusionsmaterial fehlt, hat man sich eine Verringerung der operativen Komplikationen erhofft. Eine Pseudarthrose kann, da es sich um keine fusionierende Technik handelt, nicht entstehen. Durch Erhalt einer Restbeweglichkeit im Indexsegment - also dem operierten Segment-soll Stress auf die angrenzenden Bewegungssegmente verringert werden und so die Anschlusssegmentdegeneration im Vergleich zur Spondylodese minimiert werden.

Merke

Von der dynamischen Stabilisierung erhofft man sich eine Verringerung des OP-Risikos und der Anschlusssegmentdegeneration.

Cave

Kontraindikation für alle Arten von dynamischen Stabilisierungsverfahren sind strukturelle Insuffizienzen, wie knöcherne Tumoren, Frakturen oder Osteopo-

rose.

Pedikelschrauben-basierte dynamische

Stabilisierung

Prinzip, Historie und Indikation

Das erste bekannte dynamische Stabilisierungsverfahren wurde von Henry Graf entwickelt (1992), ist als Graf-Ligamentoplastie bekannt geworden, stellt die erste PDS-Generation dar und die Basis für viele spätere Konstrukte. Über Pedikelschrauben und geflochtene Polypropylen-Bänder werden die Facettengelenke des Indexsegments in Kompression „verriegelt“ mit dem 

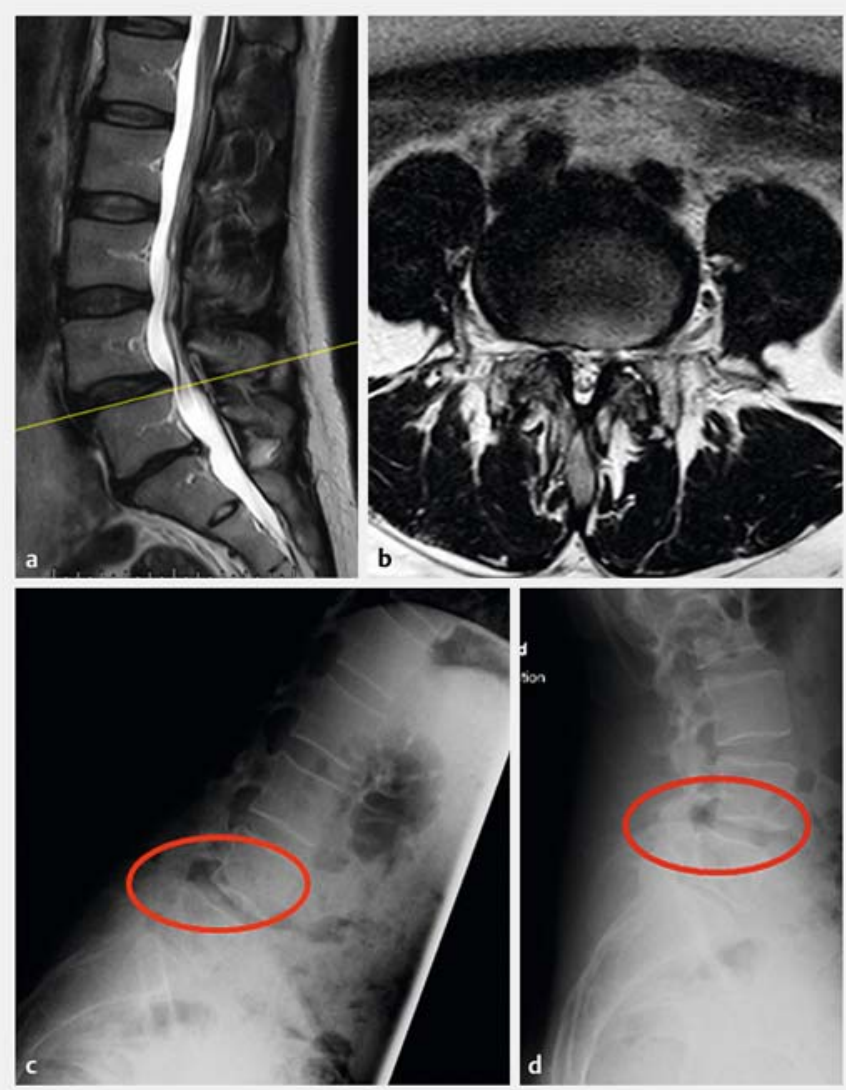

Abb. 2 a-d PDS - der typische Patient. Alter $>45$ Jahre. Rückenschmerz > Beinschmerz. Radiologische/klinische "Instabilitäts"zeichen (mono-/bisegmental). Temporäres Ansprechen auf Facettengelenkinfiltration/Thermokoagulation. Konservativ therapierefraktär.

- Tab. 2 Auswahl verschiedener PDS-Systeme.

\begin{tabular}{|c|c|c|}
\hline Produkt & Hersteller & Mechanismus der „Dynamik“ \\
\hline Graf Ligament & $\begin{array}{l}\text { Neoligaments, } \\
\text { Leeds, UK }\end{array}$ & geflochtene Polyethylen-Bänder \\
\hline Dynesis & $\begin{array}{l}\text { Zimmer Spine, } \\
\text { Minneapolis, MN }\end{array}$ & $\begin{array}{l}\text { Polyester-Bänder, Polycarbonat- } \\
\text { urethan-Spacer }\end{array}$ \\
\hline $\begin{array}{l}\text { Transition } \\
\text { Stabilisation } \\
\text { System }\end{array}$ & $\begin{array}{l}\text { Globus Medical, } \\
\text { Inc. Audubon, PA }\end{array}$ & $\begin{array}{l}\text { geflochtene Bänder, Stoßdämpfer } \\
\text { kranial der obersten Schraube, } \\
\text { flexible Spacer }\end{array}$ \\
\hline DSS & $\begin{array}{l}\text { Paradigm Spine, } \\
\text { New York, NY }\end{array}$ & dehn- und biegbare Titanwendeln \\
\hline Cosmic & $\begin{array}{l}\text { Ulrich Medical, } \\
\text { Ulm }\end{array}$ & $\begin{array}{l}\text { Scharniergelenke am Schrauben- } \\
\text { kopf }\end{array}$ \\
\hline NFlex & $\begin{array}{l}\text { Synthes, West } \\
\text { Chester, PA }\end{array}$ & $\begin{array}{l}\text { flexible Kordel und Dämpfer im } \\
\text { Titanstab }\end{array}$ \\
\hline
\end{tabular}

Ziel, dadurch eine abnormale vertikale Translation zu verhindern. Trotz einiger positiver Berichte führten die kompressionsbedingte Einengung der Neuroforamina und die zu geringe Rotationsstabilität zu unbefriedigenden Ergebnissen und erhöhten Reoperationsraten [19-21]. Eine Weiterentwicklung, das FASS-System (fulcrum-assisted soft stabilization) wurde nie klinisch eingesetzt.

Inzwischen existiert eine Vielzahl von PDS-Systemen, bei denen auf unterschiedlichste Weise eine Dynamik zwischen den Pedikelschrauben technisch umgesetzt ist ( Tab. 2).

Degenerativ bedingte Instabilität mono- oder bisegmental, meist in Kombination mit Spinalkanalstenosen oder Bandscheibenvorfällen, stellt die Hauptindikation dar. Höhergradige Spondylolisthesen (> Meyerding Grad I), Spondylolysen und längerstreckige Prozesse sollten nicht mit dem PDS versorgt werden, da diese biomechanisch dazu nicht konzipiert sind.

\section{Der „typische“ Patient}

Meist kommen ältere Patienten infrage, bei denen eine Kombination aus Stenose/Bandscheibenvorfall und segmentaler Instabilität eine Beschwerdekombination aus belastungsabhängigem Kreuzschmerz und Ischialgien (häufig pseudoradikulär) verursacht.

Typische Zeichen der Instabilität sind:

- Olisthese,

- Facettengelenkerguss,

- intradiskales Vakuumphänomen,

- Grund-/Deckplattenosteochondrose,

- provozierbarer Facettendruckschmerz,

- temporäres Ansprechen auf Facettengelenkinfiltration oder Thermokoagulation,

- Makroinstabilität in Funktionsaufnahmen (> Abb. 2.

Spondylolysen und behandlungsbedürftige Befunde über mehr als 2 Bewegungssegmente stellen Kontraindikationen zur PDS dar (> Abb. 3).

\section{Klinische Ergebnisse}

Die meisten klinischen Ergebnisse - durchweg Kohortenstudien - liegen zum Dynesis Dynamischen Neutralisations-System (Fa. Zimmer), entwickelt 1994, vor. Es besteht aus konventionellen Titan-Pedikelschrauben. Die Längsverbindung wird mit PolyethylenBändern hergestellt. Zwischen den Schraubenköpfen sitzen Distanzringe (Spacer) aus Polycarbonaturethan. Mithilfe der Bänder und Spacer lässt sich das operierte Bewegungssegment wahlweise leicht lordosieren und entlordosieren. Das Bewegungsausmaß im Indexsegment wird dadurch signifikant reduziert, tatsächlich 

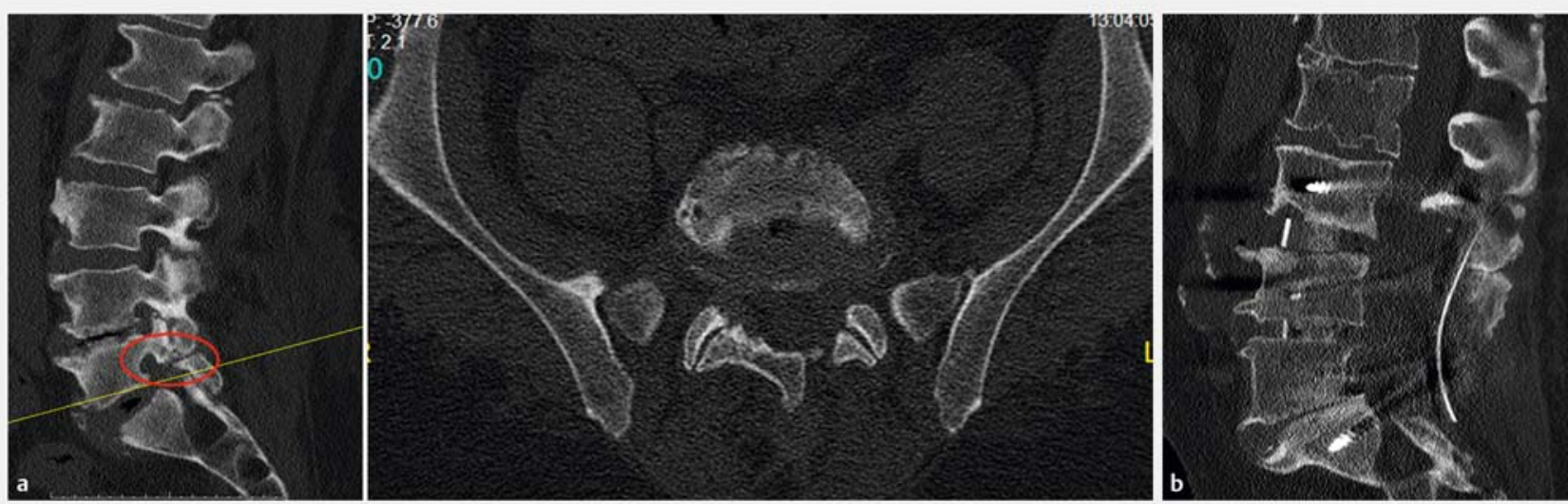

Abb. 3 Kontraindikationen zur PDS. a Lyse $\rightarrow$ rigide Stabilisierung. b Zwei Bewegungssegmente $\rightarrow$ rigide Stabilisierung.

fast so stark wie durch einen rigiden Fixateur interne [22].

Bothmann u. Mitarb. berichten in einer prospektiven Studie über 54 Patienten (Follow-up 16 Monate), denen wegen Spinalkanalstenose (41\%), DDD (28\%), Spondylolisthese Grad I (26\%) oder segmentaler Instabilität (6\%) Dynesis implantiert worden ist. Bei $81 \%$ erfolgte zusätzlich eine Nervendekompression, bei 15\% eine posteriore lumbale interkorporelle Fusion (PLIF). Schmerzen konnten signifikant reduziert werden, 72,5 $\%$ beurteilten ihr Ergebnis als gut oder exzellent. Allerdings mussten $27,5 \%$ operativ revidiert werden wegen postoperativen Komplikationen (Schraubenbruch/-Iockerung, Anschlusssegmentdegeneration) [23].

Ähnliche Revisionsraten (19\%) wurden von Grob u. Mitarb. (50 Patienten, retrospektiv, 2 Jahre Follow-up, Indikationen: DDD- und stenoseassoziierte Instabilität) berichtet. Während $67 \%$ der Patienten einen verringerten Rückenschmerz und $64 \%$ einen verringerten Beinschmerz angaben, berichteten $33 \%$ über unverändert starke oder stärkere Rückenschmerzen oder ausstrahlende Schmerzen [24]. Mögliche Erklärung dafür ist die Zunahme des Bewegungsausmaßes (ROM) im kranialen Anschlusssegment bei Krafteinwirkung nach rigidem Fixateur oder Dynesis [25]. Dynesis additiv zur Routinesequesterektomie erbrachte keinen klinischen Benefit im Langzeitverlauf von 10 Jahren [26].

Neuere Studien zeigen hingegen tendenziell bessere Ergebnisse. So erzielten Hoppe u. Mitarb. (39 Patienten, 7,2 Jahre Follow-up) nach monosegmentaler Stabilisierung $\mathrm{L} 4 / 5$ und bilateraler Dekompression bei symptomatischer degenerativer Olisthese eine recht profunde Schmerzbesserung (Rückenschmerz: 89\%, Beinschmerz: $86 \%$ ). $21 \%$ der Patienten brauchten eine weitere Operation, was bei dieser langen Nachbeob- achtungszeit auch im publizierten Rahmen nach Fusion liegt [27].

Ebenso erzielten Payer u. Mitarb. bei 30 Patienten nach monosegmentaler Dynesis-Implantation (degenerative Olisthesis und Stenose) eine profunde Verbesserung von Bein-/Rückenschmerz und ODI bis 2 Jahre nach Operation. Dabei fand sich bei 2 Patienten eine Schraubenlockerung und bei 3 Patienten eine symptomatische Anschlussdegeneration. Allerdings beobachteten die Autoren auch eine Fusionierung der Facettengelenke des Indexsegments, was einer dauerhaften Mobilität ein Ende setzt [28].

\section{FAZIT}

Zusammenfassend zeigen mehrere Dynesis-Publikationen eine hohe Rate an Schraubenlockerung oder -bruch, manche sogar eine Zunahme der präoperativen Beschwerden. Mögliche Ursachen sind die hohe Steifigkeit des Systems und die daraus resultierende Zunahme des Bewegungsumfangs im Anschlusssegment. Mittelfristig kommt es zur Facettengelenkfusion im Indexsegment. Patientenselektion scheint von großer Bedeutung zu sein.

Beim Cosmic posterioren dynamischen System (Fa. Ulrich Medical) sind die Pedikelschrauben mit einer Kalziumphosphatschicht (Bonit) beschichtet, die das Einwachsen in den Knochen fördern soll. Die Schraube ist mit dem Schraubenkopf über ein Scharniergelenk verbunden, was eine Restbeweglichkeit in Flexion und Extension ermöglicht. Biomechanische Untersuchungen haben gezeigt, dass das Bewegungsausmaß in Flexion/ Extension und Rotation dem Normalzustand ähnelt, anders als nach rigider Fixierung. Die Rotationsachse 
wandert im Vergleich zum Normalzustand nach dorsal [29].

Eine monozentrische, prospektive Beobachtungsstudie (100 Patienten, Follow-up 15 Monate) bei Patienten mit schmerzhafter degenerativer Segmentinstabilität (1-3 Höhen) mit und ohne Spinalkanalstenose berichtet über ein Patientengut, bei dem in $60 \%$ die Cosmic-Implantation nach bereits stattgehabter Voroperation erfolgt ist. Zusätzliche Dekompression erfolgte in $83 \%$. Die Verbesserung im ODI lag bei 30 (präoperativ 51, postoperativ 21), die im VAS bei 44 (präoperativ 65, postoperativ 21). Außerdem konnte die Lebensqualität (SF-36) und die Mobilität (Karnofski Performance Score) signifikant verbessert werden. Reoperationen im Follow-up mussten bei 10 Patienten wegen Schraubenlockerung $(n=2)$, persistierender Stenose/Protrusion im Indexsegment $(n=3)$, symptomatischer Anschlussdegeneration $(n=6)$ und/oder osteoporotischer Fraktur im Anschlusswirbel ( $\mathrm{n}=1$ ) erfolgen. 91 \% der Patienten waren zufrieden/sehr zufrieden. Patienten mit dynamischer Stabilisierung von mehr als 2 Segmenten mussten überproportional häufig reoperiert werden, was der zunehmenden Rigidität bei längerem Konstrukt zugeschrieben wird [30,31].

Maleci u. Mitarb. berichten über eine prospektive, multizentrische Kohorte von 139 Patienten, operiert wegen Kreuzschmerz, Ischialgie oder neurogener Klaudikation (Follow-up 2 Jahre). 70 Patienten wurden zusätzlich dekomprimiert. Sie erreichten eine Verbesserung im ODI von $48,9 \%$ auf $22,5 \%$ und im VAS von 7,3 auf 2,5 . Keine Änderung ergab sich für die lumbale Lordose und die angrenzende Bandscheibenhöhe. 11 Patienten mussten im Verlauf revidiert werden, 4 davon wegen Materialversagen. 79\% werteten das Ergebnis als exzellent/sehr gut/gut, $4 \%$ als schlecht [32].

FAZIT

Zusammenfassend existieren bisher 2 größere Kohortenstudien zur dynamischen posterioren Stabilisierung mit Cosmic. Im Vergleich zu Dynesis scheint die Rate an Materialversagen deutlich niedriger zu liegen. Es eignet sich nur für monound bisegmentalen Einsatz.

Außerdem existieren kleinere Kohortenstudien zu folgenden Systemen:

- DSS,

- NFlex,

- Accuflex,

- Bioflex Spring Rods,

- Isobar TTL.
Neben den rein dynamischen Systemen, die in jedem stabilisierten Bewegungssegment eine Restbeweglichkeit zulassen, gibt es inzwischen auch Hybridsysteme, bei denen dynamische und rigide Segmentstabilisierungen in einem Konstrukt kombiniert werden können.

Wie das Cosmic-System im direkten Vergleich zur Spondylodese bei mono- oder bisegmentaler Degeneration abschneidet, wird derzeit in einer prospektiven, randomisierten Multicenterstudie erhoben.

Bisher existiert nur Level-II-2-Evidenz zum Einsatz von Pedikelschrauben-basierten dynamischen Systemen. Bisherige Studien konnten zeigen, dass das Prinzip des Load-Sharing zwischen Bandscheibe und Facettengelenken grundsätzlich funktioniert (mono- und bisegmental), Patientenselektion aber eine wesentliche Rolle spielt. Bisher existieren nur Kurzzeitergebnisse. Verwendete Systeme sollten auch die rotatorische Instabilität reduzieren.

\section{Interspinöse Verfahren}

\section{Prinzip, Historie und Indikation}

Interspinöse Spreizer (interspinous process devices, IPD) werden über einen offenen oder perkutanen $\mathrm{Zu}$ gang in den interspinösen Raum unter das supraspinöse Band implantiert. Dabei muss die Größe so gewählt werden, dass:

- eine Distraktion der Processus spinosi,

- eine Entlastung der Facettengelenke und des hinteren Faserrings,

- eine Straffung des gelben Bandes und einer ggf. Faserringprotrusion

erfolgt.

Das führt zusätzlich zur leichten Kyphosierung des Indexsegments und zur Erweiterung der Neuroforamina.

Seit Entwicklung des ersten IPD, dem Wallis (Fa. Zimmer) 1986, ist eine Vielzahl dieser Implantate mit unterschiedlichem Design, Materialfestigkeit und Anwendungszielen auf den Markt gebracht worden. Man unterscheidet:

- flexible IPD (z. B. DIAM, Fa. Medtronic),

- semiflexible IPD (z. B. Coflex, Fa. Paradigm Spine),

- nicht flexible IPD (z. B. X-Stop, Fa. Medtronic, Wallis, Fa. Zimmer, In-Space, Fa. Synthes).

Sie wurden zur „Dekompression“ infolge oben genannter Wirkprinzipien bei ligamentär führender Spinalkanalstenose (z. B. X-Stop), zur Stabilisierung einer degenerativen Instabilität (z.B. Coflex), eines vermeintlich instabil werdenden OP-Situs nach Stenosendekompression oder Bandscheibenoperation (z.B. Wallis, DIAM) 
und zur Behandlung des Rückenschmerzes bei vermeintlichem Facettengelenksyndrom eingesetzt.

\section{Klinische Ergebnisse}

- Neurogene Klaudikation: Hierzu existieren 2 prospektive randomisierte kontrollierte Multicenterstudien (randomized controlled trial, RCT) und einige Kohortenstudien. Dabei fand sich in dem RCT von Zucherman eine signifikante Verbesserung der neurogenen Klaudikation (nach Zürich Claudicatio Questionnaire, ZCQ) im Vergleich zur konservativen Therapie bei Patienten ( $n=100$, Durchschnittsalter 70 Jahre) mit mono- oder bisegmentaler Stenose 2 Jahre nach Studieneinschluss [33]. In dem RCT von Anderson u. Mitarb. wurden 75 Patienten mit degenerativer Olisthesis und Klaudikation eingeschlossen, davon wurden 42 mit X-Stop behandelt, der Rest konservativ. Auch hier zeigte sich nach 2 Jahren eine signifikante Verbesserung der ZCQ-Werte nach $X$-Stop, nicht aber nach konservativer Therapie. Ein klinischer Erfolg (overall clinical success) trat nur bei $63 \%$ der X-Stop-Patienten und $13 \%$ der konservativ therapierten Patienten auf [34].

Jenseits der Tatsache, dass bei klinisch signifikanter Klaudikation die konservative Therapie keine adäquate Vergleichsgruppe darstellt (Equipoise-Prinzip: Gleichwertigkeit aller Studienarme mit dem Therapiestandard), zeigen die Studien wesentliche Limitationen. So ist die angewendete Blockrandomisation selektionsanfällig, das Outcome beider Studiengruppen signifikant schlechter als erwartet und die Ergebnisse eines Zentrums signifikant besser als die aller anderen.

Björn u. Mitarb. haben einen RCT $(n=100)$ zu X-Stop bei Klaudikation, die durch Flexion regredient ist, im Vergleich zur operativen Dekompression veröffentlicht (Follow-up 2 Jahre) [35]. Beide Behandlungsarme profitierten signifikant von der Therapie und zeigten keine statistischen Unterschiede nach 2 Jahren (ZCQ, VAS, SF-36). Allerdings mussten sich $26 \%$ der X-Stop-Patienten, aber nur 6\% der Dekompressionspatienten einer weiteren Operation unterziehen, was signifikant war.

- Stabilisierung nach Dekompression: Hierzu gibt es eine FDA-Zulassungsstudie (IDE) mit 322 Patienten, bei der Patienten mit Stenose und degenerativer Olisthese nach Laminektomie entweder mit Coflex interspinös stabilisiert wurden $(n=215)$ oder durch dorsolaterale Fusion und Instrumentation ( $n=107)$. Beide Gruppen zeigten eine signifikante Besserung im ODI, VAS, ZCQ. Die Coflex-Gruppe schnitt signifikant besser bez. OP-Zeit, Blutverlust, Dauer des Krankenhausaufenthalts und der ZCQ-Zufriedenheit ab. Die Fusionsgruppe musste sich seltener einer Reoperation unterziehen (14,1\% vs. 5,9\%, nicht signifikant) [36].
Zu der Arbeitshypothese, dass das Einbringen von IPDs während einer Dekompression oder Bandscheibenoperation das klinische Ergebnis verbessert, gibt es mehrere monozentrische Kohortenstudien mit geringer Patientenzahl, die keinen Vorteil durch die IPDs zeigen konnten.

Kantelhardt u. Mitarb. untersuchten 2010 die Nachhaltigkeit des IPD-Effekts und fanden, dass bereits 6 Monate nach Einbringen eines In-Space 50 \% der Patienten wieder an den präoperativen Symptomen litten [37].

\section{FAZIT}

Zusammenfassend existiert Level-I-Evidenz, dass $X$-Stop bei Claudicatio spinalis im kurzfristigen Verlauf zu einer besseren Beschwerdelinderung führt als die konservative Therapie, und dass eine Stabilisierung mit Coflex vergleichbar effektiv bei Stenose und Olisthesis ist wie die dorsolaterale Fusion, allerdings mit höherer Reoperationsrate. Zu den sonstigen Indikationen gibt es bisher keine klaren Hinweise in der Literatur, die den Nutzen der IPD beweisen. IPD sind kein nachhaltiges Verfahren.

\section{Facettengelenkersatz}

Die Rationale dieser Konstrukte entspricht im Wesentlichen der Pedikelschrauben-basierter dynamischer Systeme, der Mechanismus der „Dynamik” ahmt anatomisch die Facettengelenke nach.

Es existieren derzeit 2 Systeme:

- TOPS (total posterior solution system, Fa. Premia Spine, Israel),

- TFAS (total facet arthroplasty system, Fa. Archus Orthopaedics, Redmond, MA).

Zu TOPS wurde eine Phase-III-Studie durchgeführt, deren endgültigen Ergebnisse bisher nicht veröffentlicht wurden. Eine IDE-Studie zu TFAS wurde 2009 aus finanziellen Gründen gestoppt, hierzu existieren nur präliminäre Subgruppenergebnisse. 


\section{KERNAUSSAGEN}

- Seit das Prinzip der kompletten Stilllegung (Fusion, Spondylodese) eines degenerierten Bewegungssegments infrage gestellt worden ist, konnte die Idee der bewegungserhaltenden operativen Behandlung (Non-Fusion-Techniken) immer weiterentwickelt werden.

- Dabei stellt insbesondere die Bandscheibenprothetik (total disc replacement) eine Erfolgsgeschichte der Non-FusionsTechniken dar - wenn auch nur für ein sehr schmales Indikationsspektrum.

- Sie ist inzwischen eines der am besten untersuchten operativen Verfahren an der Wirbelsäule, deren Überlegenheit zur konservativen Therapie und zur Spondylodese in RCTs gezeigt werden konnte (Level-I-Evidenz) und das die Erwartung des nachhaltigen Bewegungserhalts erfüllen konnte.

- Der Nukleusersatz (nucleus replacement) konnte bisher trotz pathophysiologisch interessantem Konzept und einigen recht vielversprechenden Ergebnissen in klinischen Kohortenstudien (Level-II-2-Evidenz) [38] im klinischen Alltag nicht Fuß fassen.

- Bei den Pedikelschrauben-basierten dynamischen Systemen befindet man sich derzeit noch in einem präliminären Evidenzlevel. In mehreren Kohortenstudien (Level II-2) konnte nachgewiesen werden, dass das Prinzip des „load sharings” klinisch funktioniert, es allerdings teilweise auch bei dynamischen Systemen zu Fusionen im Indexsegment kommt (z. B. Dynesis).

- Das optimale Implantat und das passende Indikationsspektrum müssen noch genauer definiert werden. Außerdem steht noch der direkte Vergleich mit der Spondylodese aus.

- Hauptproblem der interspinösen Spreizer scheint die Kurzlebigkeit des klinischen Effekts zu sein

- Welche der dynamischen lumbalen Techniken die Erwartungen in Bezug auf Nachhaltigkeit und Minimierung der Anschlusssegmentdegeneration erfüllen können, wird in zukünftigen Studien zu erarbeiten sein.

\section{Interessenkonflikt}

Prof. Dr. med. Michael Stoffel hat von der Firma UIrich Medical Reisekostenunterstützung und Vortragshonorare erhalten.

\section{Über den Autor}

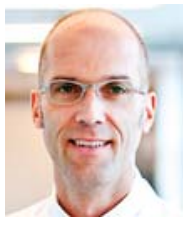

\section{Michael Stoffel}

Prof. Dr. med. Jahrgang 1968. Facharzt für Neurochirurgie. Berufliche Stationen: Klinik und Poliklinik für Neurochirurgie der Friedrich-Wilhelms-Universität Bonn, Neurochirurgische Klinik der Technischen Universität München. Seit 2011 Direktor der Neurochirurgischen Klinik, HELIOS-Klinikum Krefeld.
Sprecher der Sektion Wirbelsäule der Deutschen Gesellschaft für Neurochirurgie, Gutachter für nationale und internationale Zeitschriften.

\section{Korrespondenzadresse}

Prof. Dr. med. Michael Stoffel

HELIOS Klinikum Krefeld

Lutherplatz 40

47805 Krefeld

Phone: $02151 / 32-1320$

Fax: 02151/32-2033

E-Mail: michael.stoffel@helios-klinikum.de

Literatur

[1] Quint U, Wilke HJ. Grading of degenerative disk disease and functional impairment: imaging versus patho-anatomical findings. Eur Spine J 2008; 17: 1705-1713

[2] Schmoelz W, Onder U, Martin A et al. Non-fusion instrumentation of the lumbar spine with a hinged pedicle screw rod system: an in vitro experiment. Eur Spine J 2009; 18: $1478-1485$

[3] Fritzell P, Hägg O, Nordwall A et al. Complications in lumbar fusion surgery for chronic low back pain: comparison of three surgical techniques used in a prospective randomized study. A report from the Swedish Lumbar Spine Study Group. Eur Spine J 2003; 12: 178-189

[4] Ghiselli G, Wang JC, Bhatia NN et al. Adjacent segment degeneration in the lumbar spine. J Bone Joint Surg Am 2004; 86-A: $1497-1503$

[5] Fernström U. Arthroplasty with intercorporal endoprothesis in herniated disc and in painful disc. Acta Chir Scand 1966; $357: 154-159$

[6] Bao QB, McCullen GM, Higham PA et al. The artificial disc: theory, design and materials. Biomaterials 1996; 17: 1157 1167

[7] Ray CD. The PDN prosthetic disc-nucleus device. Eur Spine J 2002; 11 : (Suppl. 02): S137-S142

[8] Schönmayr R, Busch C, Lotz C et al. Prosthetic disc nucleus implants: the Wiesbaden feasibility study 2 years follow-up in ten patients. Riv Neuroradiol 1999; $12: 163-170$

[9] Klara PM, Rray CD. Artificial nucleus replacement: clinical experience. Spine 2002; 27: 1374-1377

[10] Bertagnoli R, Schönmayr R. Surgical and clinical results with the PDN prosthetic disc-nucleus device. Eur Spine J 2002; 11: (Suppl. 02): S143-S148

[11] Büttner-Janz K, Hochschuler SH, McAfee PC. The Artificial Disc. Berlin: Springer; 2003

[12] Braithwaite I, White J, Saifuddin A et al. Vertebral end-plate (Modic) changes on lumbar spine MRI: correlation with pain reproduction at lumbar discography. Eur Spine J 1998; 7 : 363-368

[13] Boswell MV, Colson JD, Sehgal $\mathrm{N}$ et al. A systematic review of therapeutic facet joint interventions in chronic spinal pain. Pain Physician 2007; 10: 229-253 
[14] Hellum C, Johnsen LG, Storheim K et al. Surgery with disc prosthesis versus rehabilitation in patients with low back pain and degenerative disc: two year follow-up of randomised study. BMJ 2011; 342: d2786

[15] Hellum C, Berg L, Gjertsen $\emptyset$. Norwegian Spine Study Group. et al. Adjacent level degeneration and facet arthropathy after disc prosthesis surgery or rehabilitation in patients with chronic low back pain and degenerative disc: second report of a randomized study. Spine (Phila Pa 1976) 2012; 37: $2063-2073$

[16] Jacobs W, Van der Gaag NA, Tuschel A et al. Total disc replacement for chronic back pain in the presence of disc degeneration. Cochrane Database Syst Rev 2012; 9 : CD008326

[17] Wei J, Song Y, Sun L et al. Comparison of artificial total disc replacement versus fusion for lumbar degenerative disc disease: a meta-analysis of randomized controlled trials. Int Orthop 2013; 37: 1315-1325

[18] Siepe C], Heider F, Wiechert K et al. Mid- to long-term results of total lumbar disc replacement: a prospective analysis with 5- to 10-year follow-up. Spine J 2014; 14: 1417 1431

[19] Grevitt MP, Gardner AD, Spilsbury J et al. The Graf stabilisation system: early results in 50 patients. Eur Spine J 1995; 4: $169-175$; discussion 135

[20] Markwalder TM, Wenger M. Dynamic stabilization of lumbar motion segments by use of Graf's ligaments: results with an average follow-up of 7.4 years in 39 highly selected, consecutive patients. Acta Neurochir (Wien) 2003; 145: 209-214 (discussion 214)

[21] Hadlow SV, Fagan AB, Hillier TM et al. The graft ligamentoplasty procedure: comparison with posterolateral fusion in the management of low back pain. Spine 1998; 23: 1172 1179

[22] Schmoelz W, Huber JF, Nydegger T et al. Dynamic stabilization of the lumbar spine and its effects on adjacent segments: an in vitro experiment. J Spinal Disord Tech 2003; 16: $418-423$

[23] Bothmann M, Kast E, Boldt G] et al. Dynesys fixation for lumbar spine degeneration. Neurosurg Rev 2008; 31: $189-$ 196

[24] Grob D, Benini A, Junge A et al. Clinical experience with the Dynesys semirigid fixation system for the lumbar spine: surgical and patient-oriented outcome in 50 cases after an average of 2 years. Spine (Phila Pa 1976) 2005; 30: 324 331

[25] Strube P, Tohtz S, Hoff E et al. Dynamic stabilization adjacent to single-level fusion: part I. Biomechanical effects on lumbar spinal motion. Eur Spine J 2010; 19: 2171 - 2180

[26] Hoff E, Strube P, Gross C et al. Sequestrectomy with additional transpedicular dynamic stabilization for the treatment of lumbar disc herniation: no clinical benefit after 10 years follow-up. Spine (Phila Pa 1976) 2013; 38: 887-895

[27] Hoppe S, Schwarzenbach O, Aghayev E et al. Long-Term Outcome After Monosegmental L4/5 Stabilization for Degenerative Spondylolisthesis With the Dynesys Device. Clin Spine Surg 2016; 29: $72-77$

[28] Payer M, Smoll NR, Oezkan N et al. Dynamic transpedicular stabilisation and decompression in single-level degenerative anterolisthesis and stenosis. Acta Neurochir (Wien) 2014; 156: $221-227$

[29] Bozkuş H, Senoğlu M, Baek S et al. Dynamic lumbar pedicle screw-rod stabilization: in vitro biomechanical comparison with standard rigid pedicle screw-rod stabilization. J Neurosurg Spine 2010; 12: $183-189$

[30] Stoffel M, Behr M, Reinke A et al. Pedicle screw-based dynamic stabilization of the thoracolumbar spine with the Cosmic-system: a prospective observation. Acta Neurochir (Wien) 2010; 152: 835-843

[31] Schmoelz W, Onder U, Martin A et al. Non-fusion instrumentation of the lumbar spine with a hinged pedicle screw rod system: an in vitro experiment. Eur Spine J 2009; 18: $1478-1485$

[32] Maleci A, Sambale RD, Schiavone M et al. Nonfusion stabilization of the degenerative lumbar spine. J Neurosurg Spine 2011; 15: $151-158$

[33] Zucherman JF, Hsu KY, Hartjen CA et al. A multicenter, prospective, randomized trial evaluating the X STOP interspinous process decompression system for the treatment of neurogenic intermittent claudication: two-year follow-up results. Spine (Phila Pa 1976) 2005; 30: 1351 - 1358

[34] Anderson PA, Tribus CB, Kitchel SH. Treatment of neurogenic claudication by interspinous decompression: application of the X STOP device in patients with lumbar degenerative spondylolisthesis. J Neurosurg Spine 2006; 4: 463-471

[35] Strömqvist B, Berg S, Gerdhem P et al. X-Stop versus decompressive surgery for lumbar neurogenic intermittent claudication: a randomized controlled trial with 2 years follow-up. Spine 2013; 38: 1436-1442

[36] Davis RJ1, Errico T], Bae H et al. Decompression and Coflex interlaminar stabilization compared with decompression and instrumented spinal fusion for spinal stenosis and lowgrade degenerative spondylolisthesis: two-year results from the prospective, randomized, multicenter, Food and Drug Administration Investigational Device Exemption trial. Spine (Phila Pa 1976) 2013; 38: 1529-1539

[37] Kantelhardt SR, Török E, Gempt J et al. Safety and efficacy of a new percutaneously implantable interspinous process device. Acta Neurochir (Wien) 2010; 152: 1961 - 1967

[38] U.S. Preventive Services Task Force. Guide to clinical preventive services: report of the U.S. Preventive Services Task Force. DIANE Publishing; 1989: 978-1-56806-297-6

\section{Bibliografie}

DOI http://dx.doi.org/10.1055/s-0043-102207

Die Wirbelsäule 2017; 01: 139-151

(C) Georg Thieme Verlag KG Stuttgart · New York ISSN 2509-8241 


\section{Punkte sammeln auf CME.thieme.de}

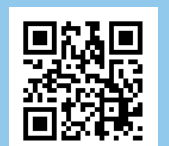

Diese Fortbildungseinheit ist 12 Monate online für die Teilnahme verfügbar.

Sollten Sie Fragen zur Online-Teilnahme haben, finden Sie unter http://cme.thieme.de/hilfe eine ausführliche Anleitung. Wir wünschen viel Erfolg beim Beantworten der Fragen!

Unter https://eref.thieme.de/ZZX8LLY oder über den QR-Code kommen Sie direkt zum Artikel zur Eingabe der Antworten.

VNR 2760512017152373652

\section{Frage 1}

Welche Aussage zu X-Stop ist falsch?

A Es gibt Evidenz für den klinischen Nutzen.

B Es gehört zu den nicht flexiblen IPD.

C Es gibt solide Langzeitergebnisse zum Einsatz bei der Klaudikation.

D Es ist bei der neurogenen Klaudikation der konservativen Therapie überlegen.

E Es kann zur leichten Kyphosierung des Indexsegments führen.

\section{Frage 2}

Welche Antwort ist falsch? Das spinale Bewegungssegment...

A besteht aus mehreren funktionellen Einheiten.

B beinhaltet u. a. die Bandscheibe.

C kann durch Degeneration symptomatisch werden.

D kann durch Höhenminderung hypermobil werden.

E beinhaltet $u$. a. die Facettengelenke.

\section{Frage 3}

Welche Aussagen zum TDR trifft zu?

A Es wird nur der Nucleus pulposus exzidiert.

B Sie hat ein breites Indikationsspektrum.

C Sie kann bei diskogenem Kreuzschmerz indiziert sein.

D Sie wird eher bei älteren Patienten ( $>50$ Jahre) eingesetzt.

E Sie kann bei therapierefraktärem Facettengelenkschmerz indiziert sein.

\section{Frage 4}

Welche Aussage zur Spondylodese trifft zu!

A Stilllegung eines Bewegungssegments ist nicht das Ziel.

B Sie verringert das Risiko einer Anschlusssegmentdegeneration.

C Die Fusionsrate entspricht der Rate an klinischem Erfolg.

D Pseudarthrosen kommen nicht mehr vor

E Je höher die operative Komplexität, desto höher die Komplikationsrate.

\section{Frage 5}

Welche Aussagen zum TDR ist falsch?

A Kinematisch werden constrained, semiconstrained und unconstrained unterschieden.

B „Facettengelenk”schmerz stellt keine Kontraindikation dar.

C Facettengelenkinfiltrationen und Diskografie haben einen diagnostischen Stellenwert.

D Laut Studienlage ist das Prothesendesign nicht entscheidend.

E Derzeit gibt es kaum Langzeitergebnisse.

\section{Frage 6}

Welche Aussage trifft nicht zu? Die dynamischen lumbalen Techniken...

A können eingeteilt werden in Gelenkprothesen und dynamische Stabilisierungen.

B werden zum Teil minimalinvasiv durchgeführt.

C sind auch bei Spondylolysen indiziert.

D können bei Kreuzschmerzen indiziert sein.

E können auch bei Vorliegen einer Spinalkanalstenose indiziert sein.

\section{Frage 7}

Welche Aussage zu den Nukleusersatzverfahren ist falsch?

A Die ersten Implantate sind häufig disloziert.

B Es wird die komplette Bandscheibe exzidiert.

C Das Wiederherstellen der Bandscheibenhöhe ist Teil des Behandlungsprinzips.

D Sie werden meist von dorsal eingebracht.

E Sie haben sich klinisch nicht durchgesetzt. 


\section{Punkte sammeln auf CME.thieme.de}

\section{Fortsetzung ...}

\section{Frage 8}

Welche Aussage zu Pedikelschrauben-basierte dynamische Stabilisierung trifft zu?

A Henry Graf ist einer der Pioniere.

B Die Graf-Ligamentoplastie kann nicht zur Neuroforameneinengung führen.

C Der typische Patient darf keine Spinalkanalstenose haben.

D Der typische Patient darf keine Spondylolisthese Meyerding I haben.

E Das Dynesis-System ist ähnlich rigide wie ein Fixateur interne.

\section{Frage 9}

Welche Aussage zur dynamischen Stabilisierung ist falsch?

A Eine Restbeweglichkeit im Bewegungssegment ist erwünscht.
B „Load Sharing” ist erwünscht.

C Sie wird nicht bei strukturellen Insuffizienzen angewandt.

D Ziel ist, die abnormale Krafteinwirkung auf Bandscheibe und Facetten zu reduzieren.

E Schellnack u. Büttner-Janz sind deren Pioniere.

\section{Frage 10}

Welche Aussage zu interspinösen Spreizern ist falsch?

A Sie wirken auch durch Distraktion des hinteren Faserrings.

B Sie können zur Kyphosierung führen.

C Sie eignen sich zur Stabilisierung nach Bandscheibenoperation oder Stenose.

D Sie werden in flexibel, semiflexibel und nicht flexibel eingeteilt.

E Sie zeichnen sich nicht durch klinische Nachhaltigkeit aus. 